\title{
Logistic Growth Model and Modeling of Factors for Community Transmission of COVID-19 in Senegal
}

\author{
Massamba Diouf ${ }^{1}$, Babacar Mbaye Ndiaye ${ }^{2}$ and Amadou Dieng ${ }^{1 *}$ \\ ${ }^{1}$ Faculty of Medicine, Pharmacy and Dentistry, Public Health Department University of Cheikh Anta Diop, \\ Senegal \\ ${ }^{2}$ Laboratory of Mathematics of Decision and Numerical Analysis University of Cheikh Anta Diop, Senegal
}

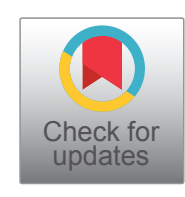

\begin{abstract}
Objective: The objective of this work is to model the variables potentially involved in the spread of cases resulting from community transmission of COVID-19 in Senegal in order to identify statistical associations.

Study design: The study carried out was of a descriptive and prospective type falling within the period from March 03, 2020 to September 20 of the same year.

Method: Firstly data on the pandemic were collected in national health system. We estimate parameters and evaluate the growth factor, community rate, weekly increase and make forecasting to help on how to find concrete actions to control the situation.

Resultat: The size will be approximately $5100 \pm 130$ cases. The discrepancy of actual and forecasted number of cases is within $3 \%$. However, actual and predicted daily new cases are scattered and vary between $26 \%$ to $600 \%$. Prediction is cumulative 3993 cases and 29 daily cases. The errors are $7.413 \%$ and $48.276 \%$ for cumulative and daily cases, respectively.

Conclusion: The pandemic in Senegal will end soon, while for most countries of the world, the blow of the anti-pandemic will be no later than the end of the year.
\end{abstract}

\section{Keywords}

COVID-19, Community case, Forecasting, Logistic model, Transmission factors.

\section{Introduction}

In December 2019, the outbreak of COVID-19 associated with a new coronavirus was a signicant event. The ensuing spread prompted the World Health Organization to declare a pandemic on March 11, 2020 after it was considered a health emergency of global concern [1]. The whole world is thus facing an unprecedented health crisis from the coronavirus with the number of people affected which is gradually increasing with more than $25,000,000$ confirmed cases for nearly 800,000 deaths at the end of August 2020 [2]. Less affected by the pandemic compared to other continents, Africa is still resisting with a COVID-19 death toll reaching nearly 30,000 deaths while confirmed cases have exceeded 1,130,000 (August 31, 2020) according to the African control center and disease prevention [3]. This progression of the disease in this zone raises concerns as well among the governors as among the health experts with regard to the living conditions of the communities, the insufficient resources to face this type of threat and the noted failures in some health systems in Africa [4-6].

In Senegal, at the start of the epidemic, the government declared a state of emergency with several health, economic and security measures taken to reduce the spread of COVID-19. Then in the face of socio-economic stress, an easing followed by the lifting of restrictive measures seems to give a boost to the disease with the meteoric increase in incident cases. From March to April, the number of cases was 933. In the period from May-June, people sick with the coronavirus increased by 5,860 . From July to August, the number of cases in Senegal increased by 6,818 This progression affecting almost all the health districts of all the regions of the country results for the most part from the resurgence of cases resulting

*Corresponding author: Amadou Dieng, Faculty of Medicine, Pharmacy and dentistry, public health department University of Cheikh Anta Diop, Dakar, Senegal

Accepted: August 27, 2021

Published online: August 29, 2021

Citation: Diouf M, Ndiaye BM, Dieng A (2021) Logistic Growth Model and Modeling of Factors for Community Transmission of COVID-19 in Senegal. J Healthcare 4(1):84-90 
from community transmission. These community cases are characterized by their difficulty in tracing to identify the source of contamination, but also by the limited possibilities for identifying and isolating the various associated contacts. During the three periods described above, the number of community cases among people positive for the coronavirus in Senegal was 109 for the first two-month period, 709 for the second two-month period and for the third two-month period July-August 2384.

This development reects an epidemiological situation including the magnitude and seriousness with regard to the consequences related to lethality and other impacts of all kinds, remain worrying. The determinants and / or variables linked to this form of transmission are not sufficiently documented in Senegal. Therefore, the identification and knowledge of the factors associated with this community transmission can constitute a decision support tool to limit the spread of the disease, on the one hand through targeted actions, to control the epidemic and its externalities on the other hand. In the previous articles [7-11], we try to forecast the pandemic for Senegal using the SIR, stochastic SIR and machine learning. In this article, we will give forecasting pandemic size of community cases for Senegal and daily predictions using the logistic model. We assume that the model is a reasonable description of the epidemic.

The objective of this work is to model the variables potentially involved in the spread of cases resulting from community transmission of COVID-19 in Senegal in order to identify statistical associations.

\section{Method}

The study carried out was of a descriptive and prospective type falling within the period from March 03, 2020 to September 20 of the same year.Full daily reports are available in [12]. We first collect data on the pandemic [13], from March 03, 2020 to September 20, 2020. Secondly, we propose transmission factors and machine learning techniques for a 2 week forecast. Variables such as the growth factor (whose formula is every day's new community cases/ new community cases on the previous day), the community rate and the weekly increase (number of cumulative cases per week) were defined to better understand the dynamics of the development of community cases in Senegal. A growth factor constant at 1 indicates there is no change in any kind of cases. When it is above 1 indicates an increase about case. When growth factor is above 1 less than but trending down ward is a positive sign. Some data analyzes followed by the Bayesian approach with a logistic model for forecasting are presented. According to the daily reports, we first analyze and preprocess the data before simulations. Variables such as the growth factor, the community rate and the weekly increase (number of cumulative cases per week) were defined to better understand the dynamics of the development of community cases in Senegal.

The numerical tests were performed by using the Python with Panda library [14] and were executed on a computer with following characteristics: Intel (R) Core-i7 CPU 2.60 GHz, 24.0
Gb of RAM, under UNIX system. According to daily reports, we first analyze and make some data preprocessing before simulations. In mathematical epidemiology, the epidemic dynamics can be described by the following variant of logistic growth model:

$$
\frac{d C}{d t}=r C\left(1-\frac{c}{k}\right)
$$

where $C$ is an accumulated number of cases, $r>0$ infection rate, and $\mathrm{K}>0$ is the final

epidemic size. If $C(0)=C_{0}>0$ is the initial number of cases then the solution of is:

$$
C=\frac{K}{1+A e^{-r t}}
$$

where $A=\frac{K-C_{0}}{{ }_{1}}$ when $t>1$, assuming $K \gg C_{0^{\prime}}$
d therefore $\mathrm{A} \gg>1 C_{0}$

We have the natural growth:

$$
C=\frac{K e^{r t}}{e^{r t}+A}=\left(\frac{C_{0}}{1-\frac{C_{0}}{K}}\right)\left(\frac{e^{r t}}{1+\frac{e^{r t}}{A}}\right) \approx C_{0} e^{r t}
$$

When $t \rightarrow \infty$ the number of community cases follows the Weibull function:

$$
C=K\left(1-A e^{-r t}+\cdots\right) \approx K\left[1+e^{-r\left(t-t_{0}\right)}\right]
$$

The growth rate $\frac{d C}{d t}$ reaches its maximum when $\frac{d^{2} C}{d^{2} t}=0$. From this condition, we obtain that the growth rate peak occurs in time:

$$
t_{P}=\frac{\ln A}{r}
$$

At this time the number of cases is

$$
C_{P}=\frac{K}{2}
$$

and the growth rate is

$$
\left(\frac{d C}{d t}\right)_{P}=\frac{r K}{4}
$$

To answer the question about doubling time $\Delta t$, i.e., the time takes to double the number

$$
\text { of cases we solve } C(t+\Delta t)=2 C(t) \text { for } \Delta t \text {. Result }
$$
is

$$
\Delta t=\frac{\ln 2}{r}-\frac{1}{r} \ln \left(\frac{1}{A}-e^{-r t}\right)-t
$$

The first term represents initial exponential growth, then $\Delta t$ increases with $\mathrm{t}$. When $t \rightarrow t_{P}=\frac{\ln A}{r}$, i.e., when 


\section{Senegal COVID-19 - Community cases}

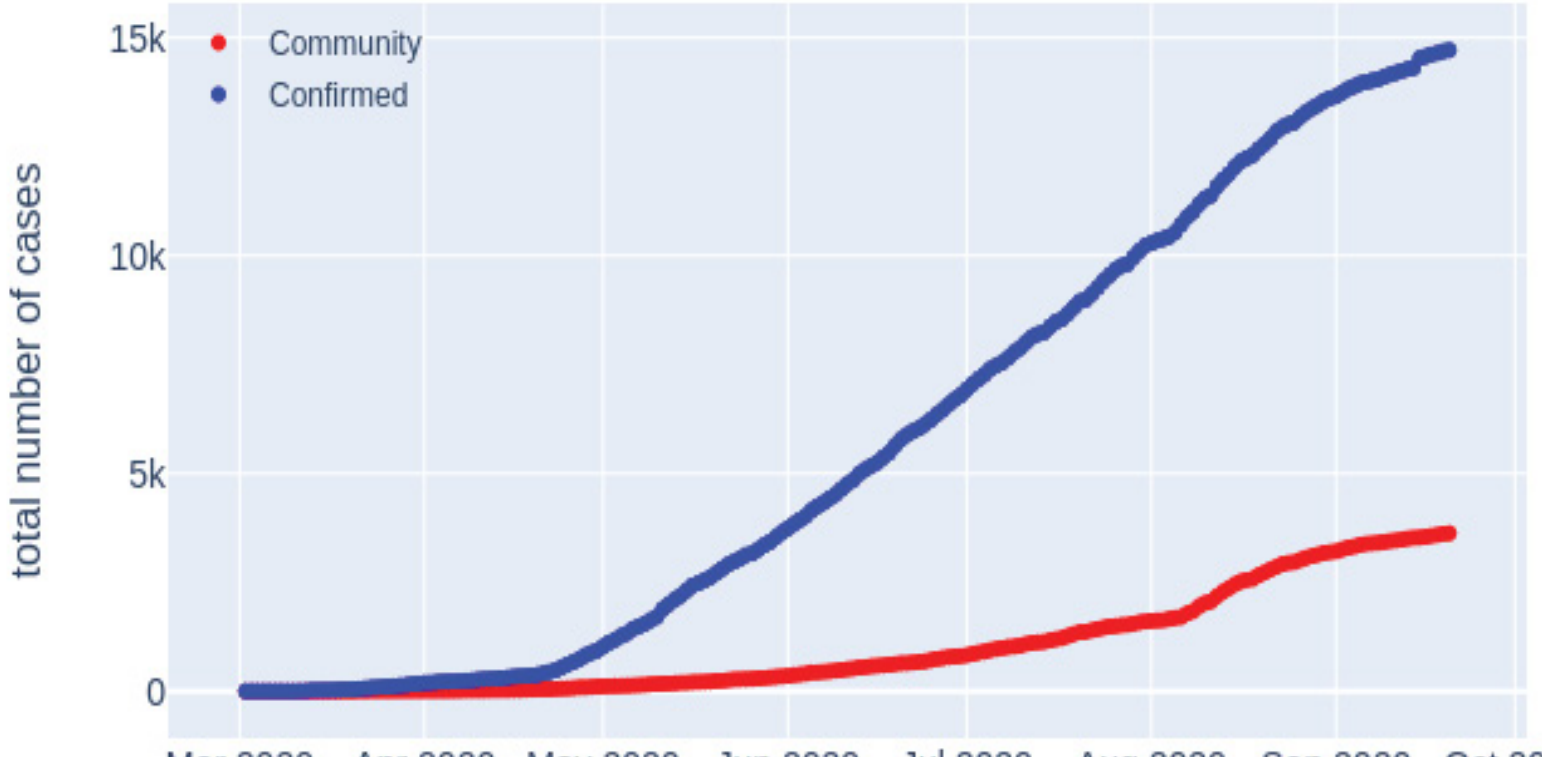

Mar 2020 Apr 2020 May 2020 Jun 2020 Jul 2020 Aug 2020 Sep 2020 Oct 2020

Figure 1: Confirmed and community cases in Senegal.

Senegal: COVID-19 community cases

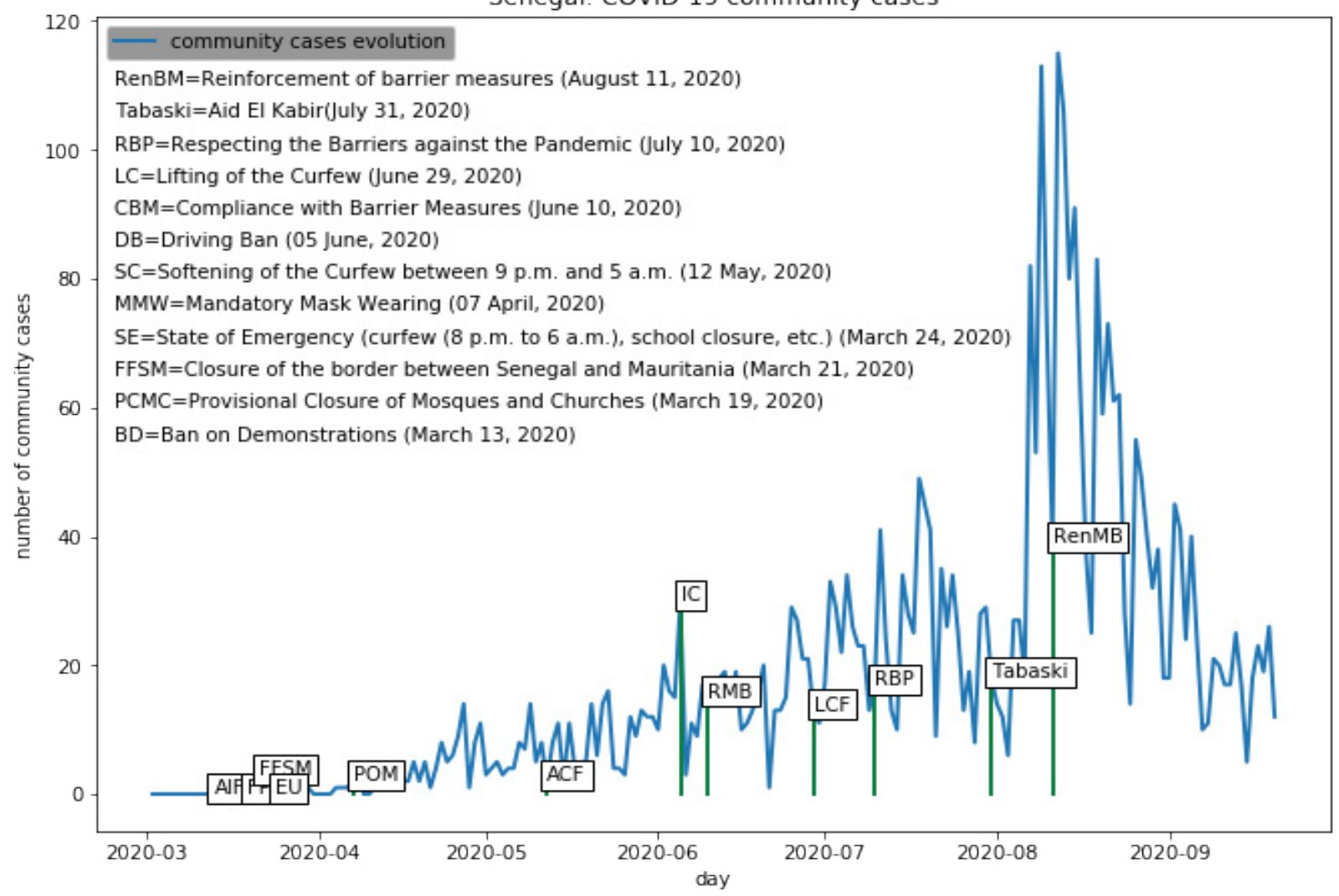

Figure 2: Community cases per day in Senegal. 
$C \rightarrow \frac{K}{2}$, then $\Delta t \rightarrow \infty$. For $C \geq \frac{K}{2}$ doubling time lost its meaning.

For more details for the logistic growth model, see [15-17].

\section{Results}

\section{Community rate}

The Figure 1 give the increase trend of confirmed cases and community cases, then Figure 2 shows the variation in the number of community cases with the various institutional measures taken since the declaration of the index Figure 3 shows a variable evolution of the rate with extreme values in April and August-September. The Figure 2 shows the dynamics of the evolution of community cases with the different institutional measures taken by the authorities and the religious or socio-cultural events leading to displacements or gatherings.

\section{Growth factor}

The growth factor is the factor by which a quantity multiplies itself over time. The growth factor for community cases is given by Figure 4, where the two largest growth peaks ( 8 and 13) are reached in May and July. The average and median growth factors are respectivly 22.56 and 17.93 .

\section{Weekly increase}

The curve and the histogram for the weekly increase in number of community cases are illustrated in Figure 5.

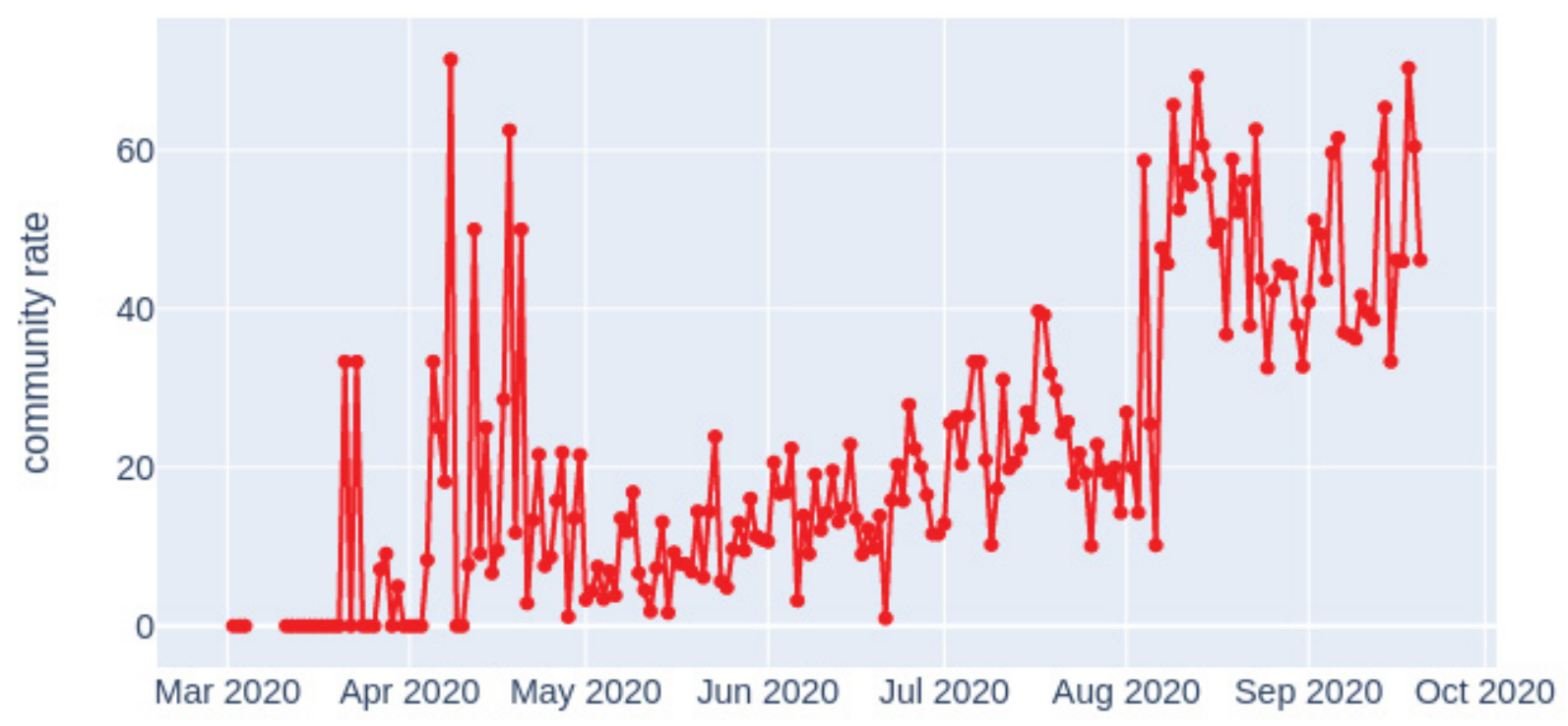

Figure 3: Community rate in Senegal.

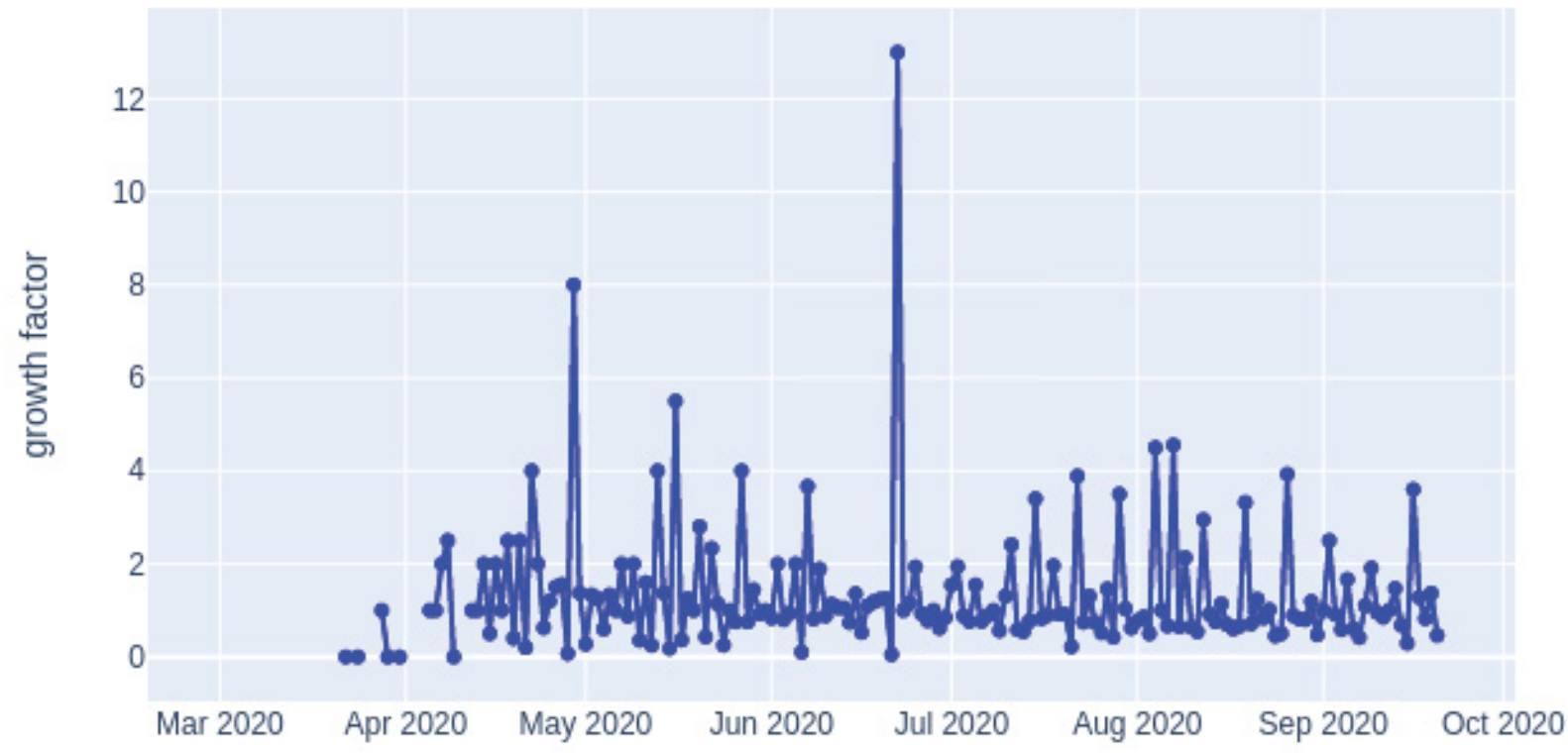

Figure 4: Growth factor per month in Senegal. 


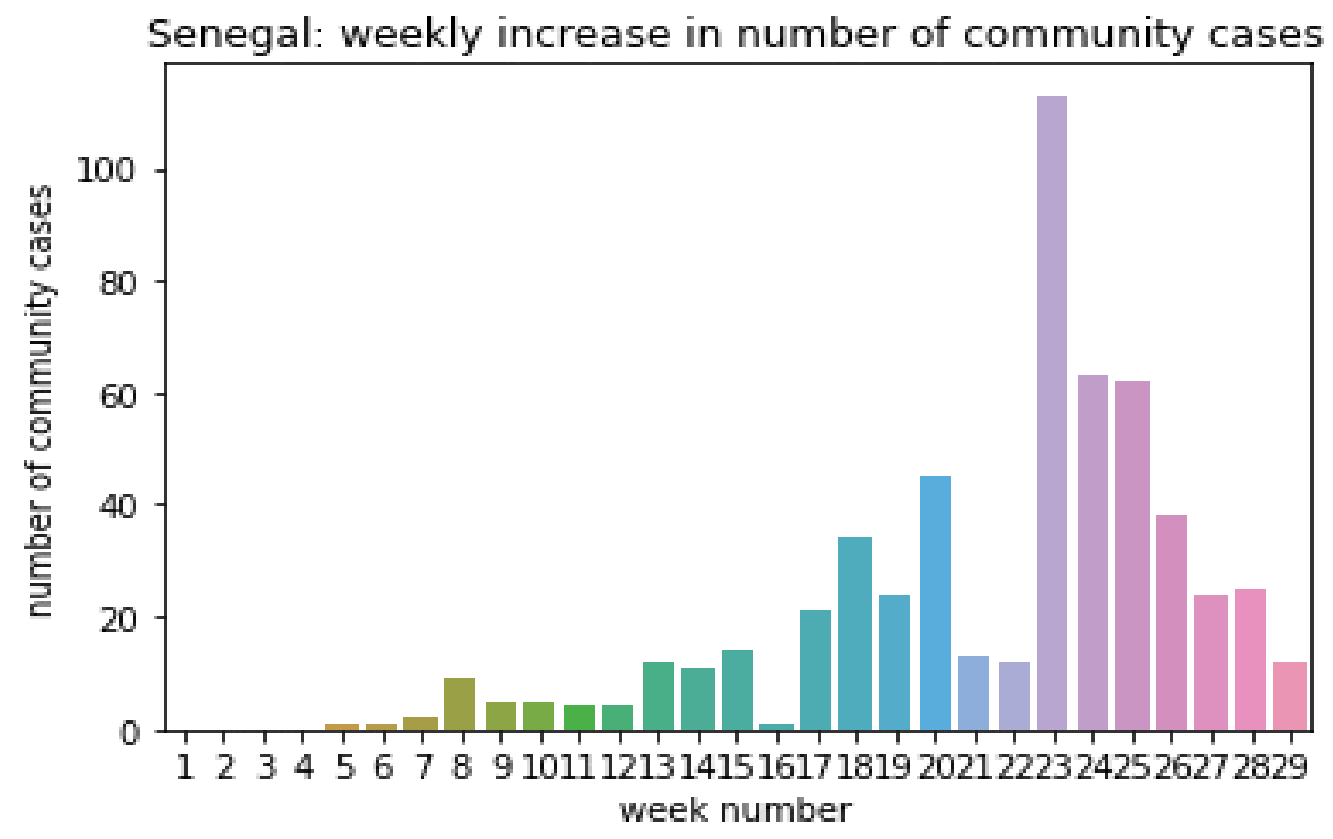

Figure 5: Growth factor per week in Senegal.

Table 1: Estimated logistic model parameters in Senegal.

\begin{tabular}{|l|l|l|l|l|}
\hline & Estimate & SE & tStat & pValue \\
\hline K & 5079.3 & 129.32 & 39.278 & $1.6383 \mathrm{e}^{-90}$ \\
\hline $\mathbf{r}$ & 0.034157 & 0.00071248 & 47.941 & $8.3375 \mathrm{e}^{-105}$ \\
\hline A & 173.03 & 10.442 & 16.571 & $3.9785 \mathrm{e}^{-38}$ \\
\hline
\end{tabular}

Number of observations: 184, Error degrees of freedom: 181

Root Mean Squared Error: 86.4

R-Squared: 0.995, Adjusted R-Squared 0.995

F-statistic vs. zero model: $2.17 \mathrm{e}+04, \mathrm{p}$-value $=3.41 \mathrm{e}^{-231}$

\section{Logisticgrow model}

We perform forecast with the logistics model [15-17] to predict the final size of coronavirus epidemy, for community cases in Senegal. The size will be approximately $5100 \pm 130$ cases (Table 1). The estimated coefficients are given in Table 2, and that the peak of the epidemic was on August 12, 2020. It seems that the epidemic in Senegal is in the ending stage (Figure 6 and Figure 7). The short-term forecasting is given in Table 2 where we see that the discrepancy of actual and forecasted number of cases is within $3 \%$. However, actual and predicted daily new cases are scattered and vary between $26 \%$ to $600 \%$. From the collected pandemic data [12], on September 25, 2020, the actual number of cases was 3697, and the daily number of cases was 15 . Prediction in Table 2 is cumulative 3993 cases and 29 daily cases. The errors are $7.413 \%$ and $48.276 \%$ for cumulative and daily cases, respectively.

\section{Discussion}

These extensive statistical analyzes allow us to make predictions about the future of the pandemic in Senegal. In the first phase of the response (at the national level), the emphasis was more on government actions, the involvement of health professionals and uncompromising communication. Then, with the increase in cases of so-called community transmission, the epidemic entered its active phase with an increasing incidence in society. This worrying situation has led to the strengthening of various measures: the state of emergency with a curfew, the prohibition or restriction of interurban travel, gatherings but above all barrier gestures such as wearing a mask. This context of restriction, like everywhere in the world, has caused a gloomy economic environment in Senegal forcing the State to readjust measures [18]. An easing of restrictions is announced first and then, a little later, the lifting of institutional measures, without first giving the necessary guarantees and support for the response strategy at the global level. Decisions that seemed to help boost the spread of the pandemic nationally with areas, initially free from the disease, which recorded their first cases of COVID-19.

In managing the pandemic, controlling movement is a sure way to limit the spread. This is why slogans urging everyone to stay at home were widely shared on social media to persuade more [19]. Despite these considerations, religious events like the Eid feast have taken place during this critical period. The festival of Eid or sacrifice of the sheep, in Senegalese sociology is a symbol and a pillar of stability and solidarity. The individuals preferentially celebrate it in family or in community. It is a time of exchanges of civilities, prayers in communion and sharing meals. This 


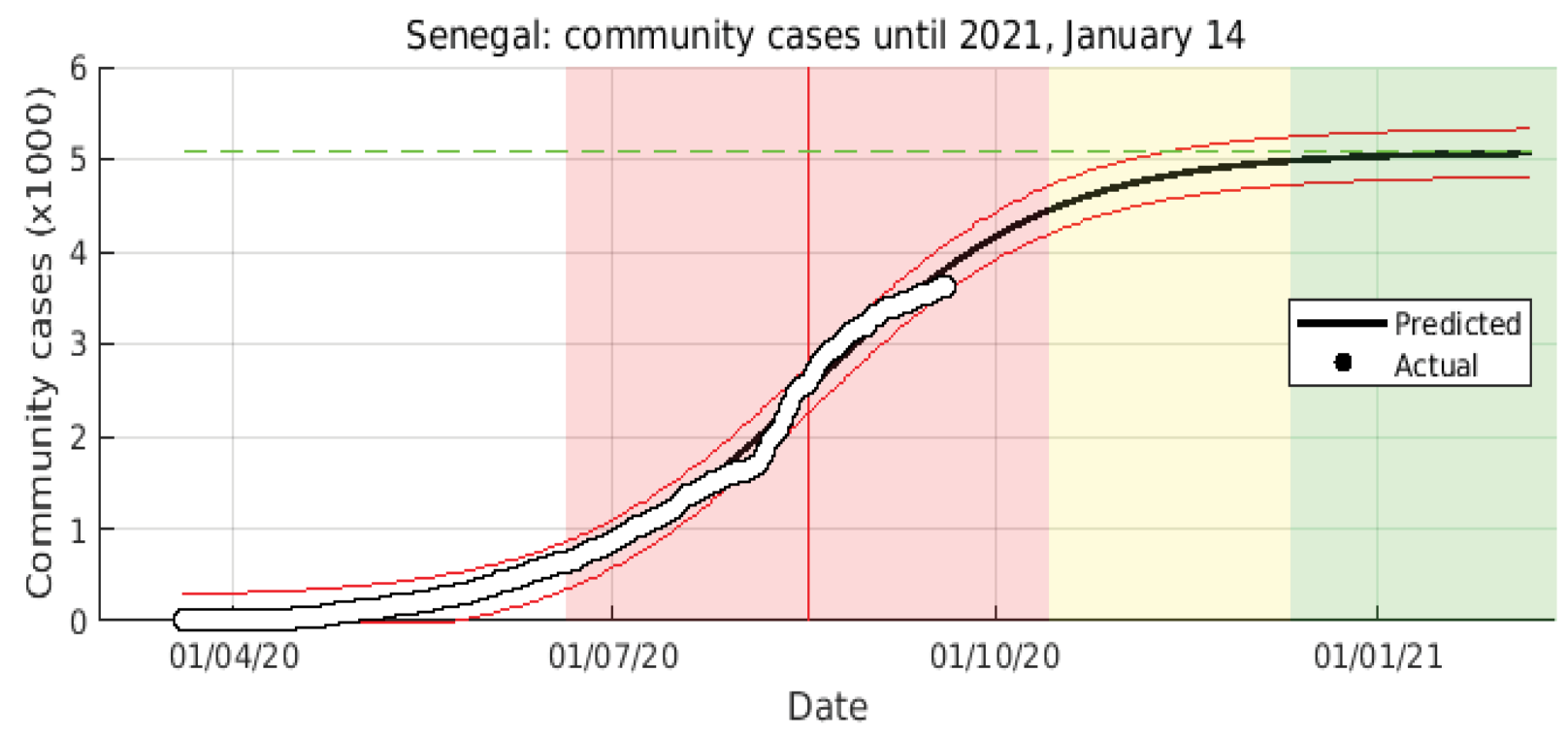

Figure 6: Logistic growth model forecasting of community cases in Senegal.

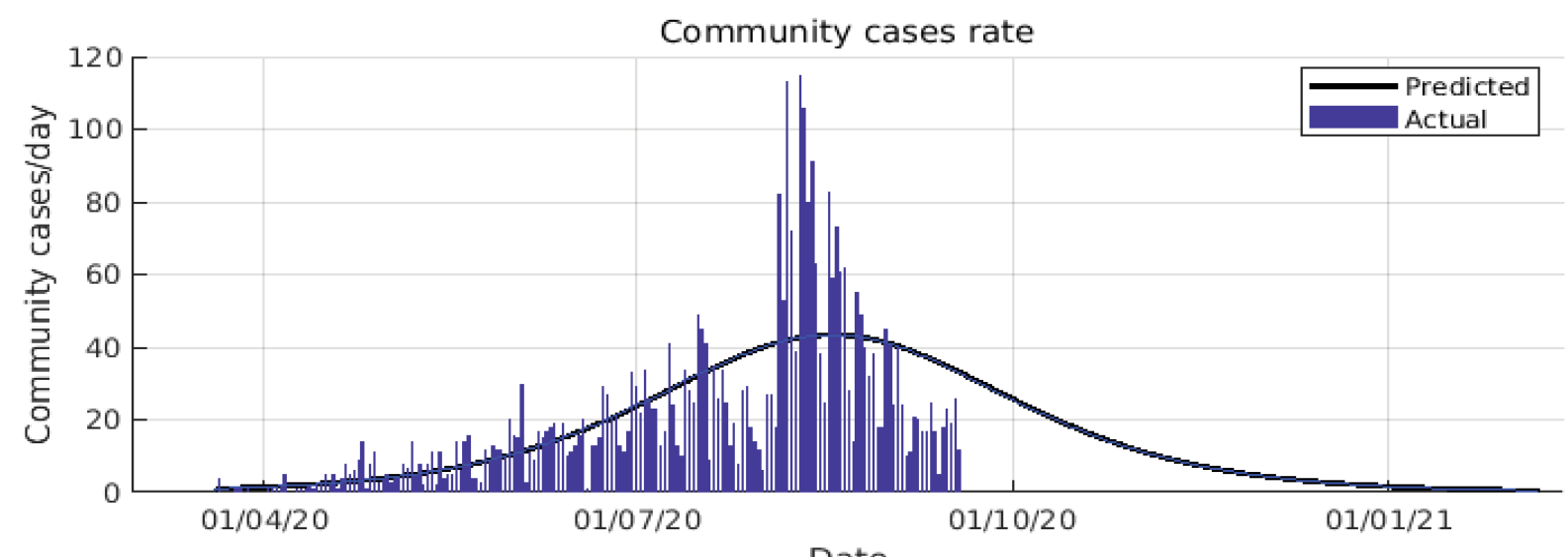

Figure 7: Forecasted final size of the community cases in Senegal.

Table 2: Short-term forecasting in Senegal.

\begin{tabular}{|c|c|c|c|c|c|c|c|}
\hline Day & Date & Actual & Predict & Error \% & Daily actual & $\begin{array}{l}\text { Daily } \\
\text { predicted }\end{array}$ & Error \% \\
\hline 179 & 14 Sept 2020 & 3527 & 3638 & 3.15 & 5 & 35 & 600.00 \\
\hline 180 & 15 Sept 2020 & 3545 & 3673 & 3.61 & 18 & 35 & 94.44 \\
\hline 181 & 16 Sept 2020 & 3568 & 3708 & 3.92 & 23 & 35 & 52.17 \\
\hline 182 & 17 Sept 2020 & 3587 & 3742 & 4.32 & 19 & 34 & 78.95 \\
\hline 183 & 18 Sept 2020 & 3613 & 3775 & 4.48 & 26 & 33 & 26.92 \\
\hline 184 & 19 Sept 2020 & 3625 & 3808 & 5.05 & 12 & 33 & 175.00 \\
\hline 185 & 20 Sept 2020 & - & 3840 & - & - & 32 & - \\
\hline 186 & 21 Sept 2020 & - & 3872 & - & - & 32 & - \\
\hline 187 & 22 Sept 2020 & - & 3903 & - & - & 31 & - \\
\hline 188 & 23 Sept 2020 & - & 3934 & - & - & 31 & - \\
\hline 189 & 24 Sept 2020 & - & 3964 & - & - & 30 & - \\
\hline 190 & 25 Sept 2020 & _ & 3993 & _ & _ & 29 & - \\
\hline
\end{tabular}


could facilitate the breaking of the barrier measures against the spread of COVID-19. Moreover, one to two weeks after the July 31 celebration of this holiday in Senegal, the rate of contamination of the disease has almost tripled with all the affected regions and the resurgence of cases resulting from community transmission.

This situation further prompted the state to make the general wearing of masks compulsory in public places, commerce, transport, schools and universities. Wearing a mask remains a beneficial measure for many countries and reduces contamination by limiting the projection of postilions (especially in public). This fact seems to contribute to the control of the disease in Senegal which, since the beginning of September, has been in a downward dynamic.

\section{Conclusion}

According to an optimistic estimate, the pandemic in Senegal will end soon, while for most countries of the world, the blow of the anti-pandemic will be no later than the end of the year. This assumes that the barrier measures and gestures can be respected. The populations must not observe a relaxation in their behavior.

\section{Acknowledgements}

We acknowledge the health statistics department of Senegal about his data support.

\section{Declaration of Competing Interest}

Authors declare that there is no conflict of interest.

\section{Author Contributions}

MD, AD: Conceptualization, Methodology, Analysis, Redaction; BMD: Conceptualization, Data Analysis, Writing, Draft preparation

\section{References}

1. World Health Organization (2019) 2019 Novel Coronavirus (2019-nCOV) strategic preparedness and response plan for south east area region.

2. World health organisation 2019 coronavirus disease outbreak.
3. Africa CDC Dashboard.

4. Coronavirus: Why is Africa globally spared from the epidemic?

5. Ray MC (2020) Cross immunity. Futura Sante.

6. Joacim Rocklov, Henrik Sjodin (2020) High population densities catalyse the spread of COVID-19. J Travel Med 1: 2.

7. Ndiaye BM, Balde MAMT, Seck D (2020) Visualization and machine learning for forecastin of COVID-19 in Senegal.

8. Ndiaye VM, Sarr SO, Ndiaye BM (2020) Impact of contamination factors on the COVID-19 evolution in Senegal.

9. Ndiaye BM, Tendeng L, Seck D (2020) Analysis of the COVID-19 pandemic by SIR model and machine learning technics for forecasting.

10. Ndiaye BM, Tendeng L, Seck D (2020) Comparative prediction of confirmed cases with COVID-19 pandemic by machine learning, deterministic and stochastic SIR models.

11. Balde MAMT, Balde C, Ndiaye BM (2020) Impact studies of nationwide measures COVID-19 anti-pandemic: Compartmental model and machine learning.

12. (2020) Ministry of health and social action.

13. Balde MAMT (2020) Fitting SIR model to COVID-19 pandemic data and comparative fore-casting with machine learning. Med Rxiv Preprint.

14. Python Software Foundation. Python Language Reference, version 2.7.

15. Wang XS, Wu JH, Yang Y (2012) Richards model revisited: Validation by and application to infection dynamics. J Theor Biol 313: 12-19.

16. Chowell SL G, Viboud C, Kuang Y (2014) Is west africa approaching a catastrophic phase oris the 2014 ebola epidemic slowing down? different models yield different answers for liberia. PLoS Curr 6.

17. Brauer F (2017) Mathematical epidemiology: Past, present, and future. Infect Dis Model 2: 113-127.

18. The heart of Senegal.

19. WHO (2019) Coronavirus disease (COVID-19) advice for the public.

DOI: $10.36959 / 569 / 468$

Copyright: (C) 2021 Diouf $M$, et al. This is an open-access article distributed under the terms of the Creative Commons Attribution License, which permits unrestricted use, distribution, and reproduction in any medium, provided the original author and source are credited. 